\title{
BAYESIAN TEXTURE AND INSTRUMENT PARAMETER ESTIMATION FROM BLURRED AND NOISY IMAGES USING MCMC
}

\author{
Cornelia VACAR, Jean-François GIOVANNELLI, Yannick BERTHOUMIEU
}

\begin{abstract}
The paper addresses an estimation problem based on blurred and noisy observations of textured images. The goal is jointly estimating the (1) image model parameters, (2) parametric point spread function (semi-blind deconvolution) and (3) signal and noise levels. It is an intricate problem due to the data model non-linearity w.r.t. these parameters. We resort to an optimal estimation strategy based on Mean Square Error, yielding the best (non-linear) estimate, namely the Posterior Mean. It is numerically computed using a Monte Carlo Markov Chain algorithm: Gibbs loop including a Random Walk Metropolis-Hastings sampler. The novelty is double: (i) addressing this fully parametric threefold problem never tackled before through an optimal strategy and (ii) providing a theoretical Fisher information-based analysis to anticipate estimation accuracy and compare with numerical results.
\end{abstract}

Index Terms - Texture, Bayes, myopic deconvolution, parameter estimation, sampling.

\section{INTRODUCTION}

The paper proposes an advance in image deconvolution, by extending the conventional estimation capabilities to textured images in a semi-blind and unsupervised context.

Deconvolution is a problem of major interest in image processing and has been widely explored (see the [1,2] overviews). The vast majority of the previous works regularize the problem through stochastic image models, such as Markov, Simultaneous Auto-Regression, Student's t, or deterministic penalties such as L2, L2-L1, L1, Total Variation (TV). These approaches use different representations: spatial domain [3,4], wavelet domain [5,6] or Fourier [7] and are essentially appropriate for piecewise-smooth and piecewiseconstant images. This limitation can be addressed using more adapted image models, this being one of our objectives.

The proposed approach considers a textured image model, based on stationary Gaussian Random Fields (GRF) [8,9].

Copyright (c) 2014 IEEE. Personal use of this material is permitted. However, permission to use this material for any other purposes must be obtained from the IEEE by sending a request to pubs-permissions@ieee.org.

C. Vacar is with the IMS Laboratory, UMR 5218, F-33400 Talence, France (e-mail: cornelia.vacar@IMS-Bordeaux.fr).

J.-F. Giovannelli is with the University of Bordeaux, IMS, UMR 5218, F-33400 Talence, France (e-mail: Giova@ IMS-Bordeaux.fr).

Y. Berthoumieu is with the Institut Polytechnique de Bordeaux and with the IMS Laboratory, UMR 5218, F-33400 Talence, France (e-mail: Yannick.Berthoumieu@IMS-Bordeaux.fr).

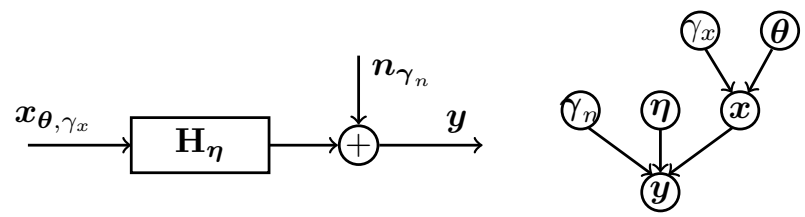

Fig. 1: Observation system and corresponding hierarchical model

This model is described by a parametric power spectral density (PSD), driven by the unknown set $\boldsymbol{\theta}$ (shape parameters) and $\gamma_{x}$ (scale parameter). This model successfully describes various texture patterns and encodes the image information in a reduced number of variables, whose estimation however is not trivial due to the cumbersome dependency.

From the instrument response viewpoint, the literature is mostly oriented towards the non-parametric point spread function (PSF), i.e., blind deconvolution [2-6, 10].

Nevertheless, in numerous applications, for instance astronomy or microscopy, the PSF is parametric, driven by the parameters $\boldsymbol{\eta}$ (myopic or semi-blind deconvolution). The paper [7] and the contributions in astronomy [11,12] tackle this problem based on a low frequency model for the image, as opposed to our textural content preserving model. The myopic deconvolution has also been applied to microscopy $[13,14]$, however, in a noiseless case, and in [15] using a TV regularization for the image, thus being primarily adapted for piecewise-smooth images.

Let us formalize our problem as:

$$
\boldsymbol{y}=\mathbf{H}_{\boldsymbol{\eta}} \cdot \boldsymbol{x}_{\gamma_{x}, \boldsymbol{\theta}}+\boldsymbol{n}_{\gamma_{n}}
$$

where $\boldsymbol{y}$ is the data and $\mathbf{H}_{\boldsymbol{\eta}}$ is the parametric PSF. $\boldsymbol{x}_{\gamma_{x}, \boldsymbol{\theta}}$ and $\boldsymbol{n}_{\gamma_{n}}$ are the unknown image and noise, both with parametric PSDs. The PSF and the image PSD are driven by $\boldsymbol{\eta}$ and $\boldsymbol{\theta}$, respectively and $\gamma_{x}$ and $\gamma_{n}$ are the hyperparameters. Fig. 1 shows the corresponding observation system and the hierarchical dependency.

A threefold problem is tackled, by jointly estimating:

1. image model parameters, $\boldsymbol{\theta}$,

2. instrument parameters, $\boldsymbol{\eta}$, (myopic or semi-blind),

3. signal and noise levels, $\gamma_{x}, \gamma_{n}$, (unsupervised), in addition to the original image $\boldsymbol{x}$. The difficulty is due to the non-linear, very intricate dependency of the data w.r.t. $\boldsymbol{\theta}$ and $\boldsymbol{\eta}$, this leading to a challenging estimation problem. To the best of our knowledge, no other method to solve this threefold deconvolution problem exists in the literature.

Moreover, for certain parameter values, the data may contain very little information, augmenting the problem dif- 
ficulty. Based on the Fisher information, a detailed analysis of the available information for each unknown is provided.

The paper is structured as follows: Section II gives the models, Section III analyses the Fisher information and Section IV details our method. Sections V and VI are devoted to the numerical results and conclusions, respectively.

\section{NOISE, TEXTURE AND PSF MODELS}

Let $N \times N$ be the image size and $P=N^{2}$ the number of pixels. $\boldsymbol{y}, \boldsymbol{x}, \boldsymbol{n} \in \mathbb{C}^{P}$ from (1) are the vectorized data, image and noise. $\boldsymbol{\Psi}=\left\{\boldsymbol{\theta}, \boldsymbol{\eta}, \gamma_{x}, \gamma_{n}\right\}$ are the unknown parameters.

The original image and the noise, $\boldsymbol{x}$ and $\boldsymbol{n}$, are modeled by independent zero-mean, stationary GRFs, with covariances $\gamma_{x} \mathbf{R}_{x}(\boldsymbol{\theta})$ and $\mathbf{R}_{n}\left(\gamma_{n}\right)$. The fields are stationary, thus the matrices have Toeplitz-block-Toeplitz structure. Moreover, for computational efficiency, a Whittle approximation is made, i.e., Circulant-block-Circulant covariance matrices, diagonalizable by Discrete Fourier Transform (DFT), computable by Fast Fourier Transform (FFT). Using a similar approximation, $\mathbf{H}_{\boldsymbol{\eta}}$ is also considered Circulant-blockCirculant. Hence, the convolution is circulant, separable in the Fourier domain:

$$
\stackrel{\circ}{y}_{p}=\stackrel{\circ}{h}_{p} \cdot \stackrel{\circ}{x}_{p}+\stackrel{\circ}{n}_{p}
$$

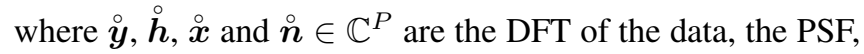
i.e., the Transfer Function (TF), the image and the noise. Let $p=1 \ldots P$ be the index of the vectorized Fourier coefficients, so that index $p$ indicates the $2 \mathrm{D}$ position $(m, n)$. To exploit the separability in the mathematical developments, the PSF, image and noise PSDs are written in the frequency domain. From (2) and the noise law Gaussianity, it follows:

$f\left(\stackrel{\circ}{p}_{p} \mid \stackrel{\circ}{x}_{p}, \boldsymbol{\eta}, \boldsymbol{\gamma}_{n}\right) \propto \mu_{p}\left(\boldsymbol{\gamma}_{n}\right) \cdot \exp \left[-\mu_{p}\left(\boldsymbol{\gamma}_{n}\right)\left|\stackrel{\circ}{y}_{p}-\stackrel{\circ}{h}_{p}(\boldsymbol{\eta}) \stackrel{\circ}{x}_{p}\right|^{2}\right]$

$$
f\left(\stackrel{\circ}{x}_{p} \mid \boldsymbol{\theta}\right) \propto \gamma_{x} \lambda_{p}(\boldsymbol{\theta}) \cdot \exp \left[-\gamma_{x} \lambda_{p}(\boldsymbol{\theta})\left|\stackrel{\circ}{x}_{p}\right|^{2}\right]
$$

where $\mu_{p}\left(\boldsymbol{\gamma}_{n}\right), \lambda_{p}(\boldsymbol{\theta})$ and $\stackrel{\circ}{h}_{p}(\boldsymbol{\eta})$ are the eigenvalues of $\mathbf{R}_{n}$, $\mathbf{R}_{x}$ and $\mathbf{H}_{\boldsymbol{\eta}}$.

An important point is the method adaptedness to any TF and any PSD for image and noise. However, for the mathematical illustration and numerical evaluation, we chose:

- TF - low pass filter (Dirichlet kernel), of width $\eta$ :

$$
\stackrel{\circ}{\boldsymbol{h}}\left(\nu_{x}, \nu_{y}, \boldsymbol{\eta}\right)=\frac{1}{2 \boldsymbol{\eta}} \frac{\sin \left(2 \pi \boldsymbol{\eta} \sqrt{\nu_{x}^{2}+\nu_{y}^{2}}\right)}{\sin \left(\pi \sqrt{\nu_{x}^{2}+\nu_{y}^{2}}\right)}
$$

- noise - white noise of covariance $\mathbf{R}_{n}\left(\gamma_{n}\right)=\gamma_{n}^{-1} \mathbf{I}$, with $\gamma_{n}$ precision parameter.

- image - exponential model for the PSD:

$$
\boldsymbol{\lambda}^{-1}\left(\nu_{x}, \nu_{y}, \boldsymbol{\theta}\right)=\exp \left[-\frac{\left|\nu_{x}-\nu_{x}^{0}\right|}{u_{x}}-\frac{\left|\nu_{y}-\nu_{y}^{0}\right|}{u_{y}}\right]
$$

with $\boldsymbol{\theta}=\left[\nu_{x}^{0}, \nu_{y}^{0}, u_{x}, u_{y}\right] \in \mathbb{R}^{4}$. The parameters $\nu_{x}^{0}, \nu_{y}^{0}$ are the central frequencies, $u_{x}, u_{y}$ are the PSD widths.
The variables $\nu_{x}, \nu_{y} \in[-0.5,0.5]^{2}$ are the continuous reduced frequencies, while $\left(\nu_{m}, \nu_{n}\right)$ are the discretized reduced frequencies. We associate the frequency pair $\left(\nu_{m}, \nu_{n}\right)$ to coefficient $p$. Then $\lambda_{p}(\boldsymbol{\theta})=\boldsymbol{\lambda}^{-1}\left(\nu_{m}, \nu_{n}, \boldsymbol{\theta}\right)$.

In (5) and (6), the dependency of $\stackrel{\circ}{h}_{p}$ and $\lambda_{p}$ w.r.t. the unknown parameters $\boldsymbol{\eta}$ and $\boldsymbol{\theta}$ is highly non-linear and intricate, this representing the main problem difficulty.

\section{INFORMATION QUANTITATIVE ASSESSMENT}

The data may carry different amounts of information, this affecting directly the estimation performance, depending on the Signal to Noise Ratio (SNR $=\gamma_{n} / \gamma_{x}$ ) and the parameter values. The mean available information on $\boldsymbol{\Psi}$ is quantified by the Fisher information matrix. We focus on each parameter $\psi$ (any one of $\boldsymbol{\Psi}$ elements), through its diagonal elements, i.e., the expectation of the log-likelihood second derivative:

$$
\mathcal{I}(\psi)=-\mathbb{E}_{\boldsymbol{y} \mid \Psi}\left[\frac{\partial^{2}}{\partial \psi^{2}} \log f(\boldsymbol{y} \mid \Psi)\right]
$$

$f(\boldsymbol{y} \mid \boldsymbol{\Psi})$ is obtained from (1), (3) and (4) and writes: $\boldsymbol{y} \mid \boldsymbol{\Psi} \sim$ $\mathcal{N}\left(0, \mathbf{R}_{y}(\boldsymbol{\Psi})\right)$, with $\mathbf{R}_{y}(\boldsymbol{\Psi})=\gamma_{x} \mathbf{H}_{\boldsymbol{\eta}} \mathbf{R}_{x}(\boldsymbol{\theta}) \mathbf{H}_{\boldsymbol{\eta}}^{\mathrm{t}}+\mathbf{R}_{n}\left(\gamma_{n}\right)$. The matrix $\mathbf{R}_{y}(\boldsymbol{\Psi})$ is Circulant-block-Circulant, due to the form of $\mathbf{H}_{\boldsymbol{\eta}}, \mathbf{R}_{x}(\boldsymbol{\theta})$ and $\mathbf{R}_{n}\left(\gamma_{n}\right)$. Thus, it is diagonalizable by DFT, with entries $r_{p}(\boldsymbol{\Psi})$, namely the variance of $\stackrel{\circ}{p}_{p} \mid \Psi$ :

$$
r_{p}(\boldsymbol{\Psi})=g_{p}(\boldsymbol{\eta}) \gamma_{x}^{-1} \lambda_{p}^{-1}(\boldsymbol{\theta})+\gamma_{n}^{-1}
$$

where $g_{p}(\boldsymbol{\eta})=\left|\stackrel{\circ}{h}_{p}(\boldsymbol{\eta})\right|^{2}$.

In (7), since $\mathbb{E}_{\boldsymbol{y} \mid \psi}\left[\left|\dot{y}_{p}\right|^{2}\right]=r_{p}(\boldsymbol{\Psi})$, the second order derivatives cancel out when taking the expectation. Thus:

$$
\mathcal{I}(\psi)=\sum_{p}\left[\frac{1}{r_{p}(\mathbf{\Psi})} \cdot r_{p}^{\prime}(\mathbf{\Psi})\right]^{2}
$$

$r_{p}^{\prime}$ being the derivative of $r_{p}$ w.r.t. $\psi$. Then, regarding $\gamma_{n}$ :

$$
\mathcal{I}\left(\gamma_{n}\right)=\gamma_{n}^{-2} \sum_{p}\left[1+\gamma_{n} / \gamma_{x} \cdot g_{p}(\boldsymbol{\eta}) \lambda_{p}^{-1}(\boldsymbol{\theta})\right]^{-2}
$$

It is a decreasing function, hence, the smaller the $\gamma_{n}$ (the higher the noise level), the easier its estimation. Similarly, $\mathcal{I}\left(\gamma_{x}\right)$ is also a decreasing function.

For a texture parameters $\theta$, element of $\boldsymbol{\theta}$ :

$$
\mathcal{I}(\theta)=\sum_{p}\left\{\frac{g_{p}(\boldsymbol{\eta}) \cdot \lambda_{p}^{\prime}(\boldsymbol{\theta})}{\lambda_{p}(\boldsymbol{\theta})\left[\gamma_{x} / \gamma_{n} \cdot \lambda_{p}(\boldsymbol{\theta})+g_{p}(\boldsymbol{\eta})\right]}\right\}^{2}
$$

As expected, the lower the SNR, the smaller each term, thus, the less information on $\theta$. For a central frequency $\theta=\nu^{0}$, the information is higher when:

- $\nu^{0}$ is close to 0 , due to the low-pass filter,

- the corresponding width is small.

For a width $\theta=u, \mathcal{I}(u)$ is higher when:

- $u$ is smaller,

- the corresponding $\nu^{0}$ is closer to 0 .

Another interesting case is the noiseless one $\left(\gamma_{n}=\infty\right)$ : $\mathcal{I}(\theta)=\sum_{p}\left[\lambda_{p}^{\prime}(\boldsymbol{\theta}) / \lambda_{p}(\boldsymbol{\theta})\right]^{2}$. The information on $\theta$ depends 


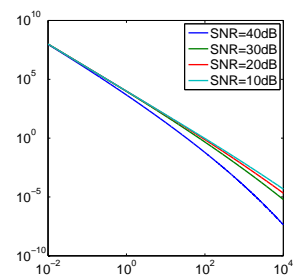

(a) $\mathcal{I}\left(\gamma_{n}\right)$

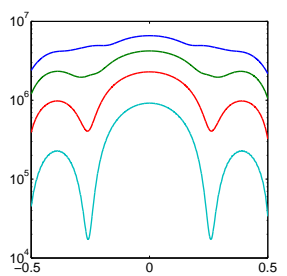

(b) $\mathcal{I}\left(\nu^{0}\right)$

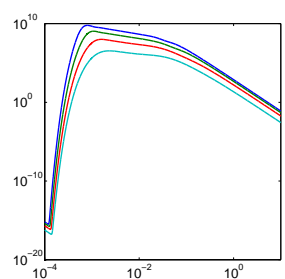

(c) $\mathcal{I}(u)$
Fig. 2: Fisher information, in logarithmic scale, for the noise level $\gamma_{n}$, a PSD central frequency $\nu^{0}$ and a PSD width $u$.

only on the texture model and its parameters, but not at all on the TF (when the TF is known).

The same considerations hold for the TF parameter $\boldsymbol{\eta}$ :

$$
\mathcal{I}(\boldsymbol{\eta})=\sum_{p}\left[\frac{g_{p}^{\prime}(\boldsymbol{\eta})}{\gamma_{x} / \gamma_{n} \cdot \lambda_{p}(\boldsymbol{\theta})+g_{p}(\boldsymbol{\eta})}\right]^{2}
$$

For low SNR, $\mathcal{I}(\boldsymbol{\eta})$ is small and, in the noiseless case, $\mathcal{I}(\boldsymbol{\eta})$ only depends on the TF, with $\mathcal{I}(\boldsymbol{\eta})=\sum_{p}\left[g_{p}^{\prime}(\boldsymbol{\eta}) / g_{p}(\boldsymbol{\eta})\right]^{2}$ (when the PSD is known).

Fig.2 illustrates the evolution of the Fisher information for $\gamma_{n}, \nu^{0}$ and $u$, for four SNR levels. A more quantitative analysis of $\mathcal{I}\left(\gamma_{n}\right)$ from (10), for instance, can be done from Fig.2a: we observe that $\mathcal{I}\left(10^{-2}\right)=4 \cdot 10^{7}$, while $\mathcal{I}\left(10^{-1}\right)=$ $4 \cdot 10^{5}$, meaning that the gain of an order of magnitude for the noise level implies the gain of two orders of magnitude in information amount. Consequently, we should expect the same trend in the numerical results.

Different scenarios are illustrated by Fig.3, as 1D crosssections of the 2D frequency domain:

(a) for narrow TFs (small $\boldsymbol{\eta}$ ) and high frequency PSDs (large $\left(\nu_{x}^{0}, \nu_{y}^{0}\right)$ ), the spectral contents cancel each other, i.e., the data is informative on $\gamma_{n}$, but not on $\boldsymbol{\eta}$ and $\boldsymbol{\theta}$;

(b) for wide TFs and narrow PSDs, the input stimulus is incapable to induce an adequate system perturbation, i.e., the information is insufficient to estimate $\boldsymbol{\eta}$;

(c) ideal situation information-wise, i.e., partial overlap. The information available for the estimation depends on this overlap.

Section $\mathrm{V}$ provides a numerical evaluation of the method performances, related to the Fisher information study.

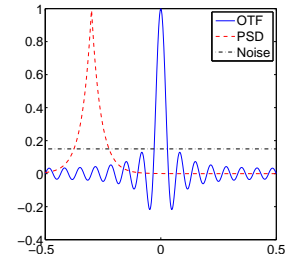

(a) Narrow TF

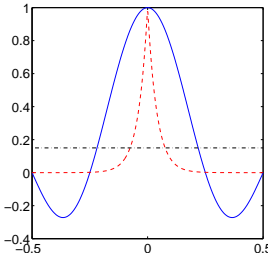

(b) Wide $\mathrm{TF}$

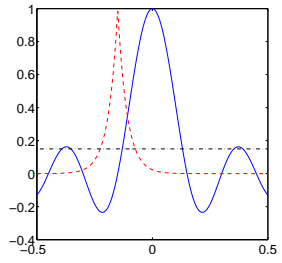

(c) Partial overlap
Fig. 3: Different relative positioning and widths for TF and PSD. On the abscissa, the reduced frequency domain is represented.

\section{BAYESIAN FORMULATION}

The addressed problem is challenging since it consists in jointly estimating the PSD and TF parameters, the hyperparameters and, in addition, the image. This problem is tackled in a Bayesian approach, all the unknowns being probabilized and assigned a prior. Let us denote $\pi(\boldsymbol{\Psi})$ the prior for $\boldsymbol{\Psi}$.

From Bayes rule and Fig.1, the joint law writes:

$$
\pi(\boldsymbol{y}, \boldsymbol{x}, \Psi)=f\left(\boldsymbol{y} \mid \boldsymbol{x}, \boldsymbol{\eta}, \gamma_{n}\right) \cdot f(\boldsymbol{x} \mid \boldsymbol{\theta}) \cdot \pi(\boldsymbol{\Psi})
$$

and the posterior law is proportional to the joint law:

$$
\begin{aligned}
& \pi(\boldsymbol{x}, \boldsymbol{\Psi} \mid \boldsymbol{y}) \propto \gamma_{n}^{P} \exp \left[-\gamma_{n} \sum_{p}\left|\stackrel{\circ}{y}_{p}-\stackrel{\circ}{x}_{p} \stackrel{\circ}{h}_{p}(\boldsymbol{\eta})\right|^{2}\right] \\
& \cdot \gamma_{x}^{P} \cdot \prod_{p} \lambda_{p}(\boldsymbol{\theta}) \cdot \exp -\left[\gamma_{x} \sum_{p} \lambda_{p}(\boldsymbol{\theta})\left|\stackrel{\circ}{x}_{p}\right|^{2}\right] \cdot \pi(\boldsymbol{\Psi})
\end{aligned}
$$

We are considering a situation of poor available information about the parameters, not indicating prior dependency between the unknowns. We thus use a separable prior, i.e., $\pi(\boldsymbol{\Psi})=\pi(\boldsymbol{\theta}) \cdot \pi(\boldsymbol{\eta}) \cdot \pi\left(\gamma_{x}\right) \cdot \pi\left(\gamma_{n}\right)$, each factor being diffuse.

The forms for the prior laws can be judiciously chosen by analyzing (14). It can thus be noticed that $\gamma_{x}$ and $\gamma_{n}$ intervene as inverse variance parameters in a Gaussian law, hence the Gamma law is a conjugate form:

$$
\pi(\gamma \mid \alpha, \beta) \propto \gamma^{\alpha-1} \exp -\beta \gamma
$$

Furthermore, since we have very little prior information on these parameters, uninformative Jeffreys priors is used, by setting $(\alpha, \beta) \rightarrow(0,0)$.

For $\boldsymbol{\theta}$ and $\boldsymbol{\eta}$, the dependency of the likelihood w.r.t. the parameters is very complicated, meaning that there is no conjugate form. Moreover, the lack of prior information suggests the use of the uniform law:

$$
\pi(\boldsymbol{\theta})=\mathcal{U}_{\left[\boldsymbol{\theta}_{m}, \boldsymbol{\theta}_{M}\right]}(\boldsymbol{\theta}) \quad \pi(\boldsymbol{\eta})=\mathcal{U}_{\left[\boldsymbol{\eta}_{m}, \boldsymbol{\eta}_{M}\right]}(\boldsymbol{\eta})
$$

Remark: The uninformative priors might give the wrong impression that our ill-posed problem is not regularized. In fact, this is done through highly structured models for the TF and image and not through the priors on $\Psi$.

The Posterior Mean (PM) estimator is chosen due to its mean square error (MSE) optimality. It consists in calculating the integral $\int_{\boldsymbol{\Psi} \mid \boldsymbol{y}} \boldsymbol{\Psi} \pi(\boldsymbol{\Psi} \mid \boldsymbol{y}) \mathrm{d} \boldsymbol{\Psi}$. The complicated parameter dependency makes it intractable, thus numerical methods are required. Among the various options, we chose stochastic sampling, more specifically expectation approximation by empirical mean of posterior samples. Since direct joint sampling is impossible, we use a Gibbs loop consisting in iterative conditional sampling.

Remark: In this context, $\boldsymbol{x}$ could be marginalized to avoid its sampling, but this would lead to complicated laws for the other parameters and thus to increased sampling complexity.

The conditional law for the image is Gaussian and separable in the Fourier domain, a key feature of the proposed 


\begin{tabular}{crccccccc} 
SNR & TF & $\gamma_{n}$ & $\gamma_{x}$ & $\nu_{x}^{0}$ & $\nu_{y}^{0}$ & $u_{x}$ & $u_{y}$ & $\boldsymbol{\eta}$ \\
\hline \multirow{2}{*}{$20 \mathrm{~dB}$} & Narrow & 1.2 & 3.2 & 4 & 4.2 & 8.5 & 8 & 4.3 \\
& Wide & 2.5 & 1.9 & 3 & 3.1 & 6.3 & 6.5 & 12 \\
& Overlap & 1.5 & 1.1 & 1.2 & 1.3 & 3.4 & 3.5 & 3 \\
\hline \multirow{2}{*}{$15 \mathrm{~dB}$} & \multirow{2}{*}{ Overlap } & 3.2 & 10.8 & 12.5 & 11.8 & 15.9 & 17.3 & 14.5 \\
$25 \mathrm{~dB}$ & & 1.9 & 1.6 & 1.1 & 0.9 & 3.1 & 3 & 2.7 \\
\hline
\end{tabular}

Table I: Parameter estimation MSE - performance in various scenarios, such as those in Fig.3, and for various SNR levels.

method, since it allows fast parallel sampling of the image Fourier coefficients. The hyperparameters have Gamma conditional laws, thus sampling is straightforward. However, $\boldsymbol{\theta}$ and $\boldsymbol{\eta}$ have non-standard laws, thus more complex sampling strategies must be used. We chose to include a Random-Walk Metropolis-Hastings (RWMH) step within the Gibbs loop. This strategy is convergent since i) the Metropolis within Gibbs sampler provides samples from the posterior law [16] and ii) the empirical mean of these samples converges to the posterior expectation. The RWMH jump amplitude is tuned so that the acceptance rate is approximately $24 \%$ [17].

Our algorithm then consists of:

1. Initialization phase: $\boldsymbol{\theta}^{(1)}, \boldsymbol{\eta}^{(1)}, \gamma_{x}^{(1)}, \gamma_{n}^{(1)}, i=1$

2. For $i=2,3 \ldots$

$\stackrel{\circ}{x}_{p}^{(i)} \sim \mathcal{N}\left(\gamma_{n}^{(i-1)} \stackrel{\circ}{h}_{p}\left(\boldsymbol{\eta}^{(i-1)}\right) \stackrel{\circ}{y}_{p} \cdot \sigma_{p}, \quad \sigma_{p}^{2}\right) \quad$ in parallel $\forall p$

with $\sigma_{p}=\left[\gamma_{n}^{(i-1)} g_{p}\left(\boldsymbol{\eta}^{(i-1)}\right)+\gamma_{x}^{(i-1)} \lambda_{p}\left(\boldsymbol{\theta}^{(i-1)}\right)\right]^{-1}$

$\gamma_{n}^{(i)} \sim \mathcal{G}\left(\alpha+P, \beta_{n}^{\text {post }}\right) \quad \gamma_{x}^{(i)} \sim \mathcal{G}\left(\alpha+P, \beta_{x}^{\text {post }}\right)$

with $\beta_{n}^{\text {post }}=\beta+\sum_{p}\left|\stackrel{\circ}{y}_{p}-\stackrel{\circ}{x}_{p}^{(i)} \stackrel{\circ}{h}_{p}\left(\boldsymbol{\eta}^{(i-1)}\right)\right|^{2}$

$$
\beta_{x}^{\text {post }}=\beta+\sum_{p} \lambda_{p}\left(\boldsymbol{\theta}^{(i-1)}\right)\left|\stackrel{\leftrightarrow}{x}_{p}^{(i)}\right|^{2}
$$

$\boldsymbol{\theta}^{(i)} \sim$ RWMH step with target $\pi(\boldsymbol{\theta}) \propto f\left(\boldsymbol{x}^{(i)} \mid \gamma_{x}^{(i)}, \boldsymbol{\theta}\right)$

$\boldsymbol{\eta}^{(i)} \sim$ RWMH step with target $\pi(\boldsymbol{\eta}) \propto f\left(\boldsymbol{y} \mid \boldsymbol{x}^{(i)}, \boldsymbol{\eta}, \gamma_{n}^{(i)}\right)$

Remark: The conditional law of the image has as maximizer the image obtained through Wiener filtering. In the noiseless case, the maximizer is obtained by inverse filtering.

The stopping condition is that the variations of the recursive empirical mean from one iteration to the next are less than $0.1 \%$ for all the parameters.

The algorithm requires only two Fourier transforms, at the beginning and at the end, all the intermediary computations being done in the Fourier domain, in parallel. This makes the algorithm very efficient, the running time being less than one minute for $256 \times 256$ size images, for the roughly $2 \cdot 10^{3}$ iterations needed to reach convergence and compute the PM.

\section{RESULTS}

The tests were performed on 20 realizations of each scenario (narrow TF, wide TF, partial overlap) for different parameter values. The SNR quantifies the original signal to

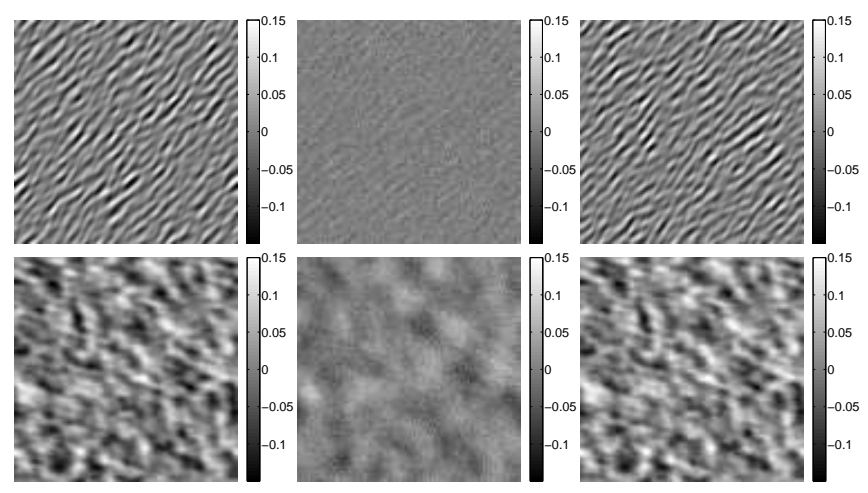

Fig. 4: Examples of original image, corresponding blurred and noisy observations and the deconvolution results.

noise ratio, nevertheless, the Blurred SNR (used in [3] to quantify the problem difficulty) is significantly smaller and depends on the relative positioning of the TF and PSD.

Table I lists the mean relative error (\%). The data is more or less informative depending on the parameter (see Section III), and this explains the estimation error difference. As anticipated by the Fisher information in Fig.2, the $\left\{\nu_{x}^{0}, \nu_{y}^{0}\right\}$ are better estimated as compared to $\left\{u_{x}, u_{y}\right\}$ and $\boldsymbol{\eta}$. Moreover, the estimation accuracy increases with the SNR for all the parameters, except $\gamma_{n}$. However, for too low SNR, even the $\gamma_{n}$ error is high, due to the higher estimation errors for the other parameters. We have also tested the method in a partial overlap configuration and SNR $=25 \mathrm{~dB}$, when $\gamma_{n}^{*}=10^{-1}$ and $\gamma_{n}^{*}=10^{-2}$. The estimation errors for $\gamma_{n}$ were $2.3 \%$ and $1.9 \%$, respectively, coherent with the considerations on the available information in Section III.

Moreover, we must stress that, despite the problem difficulty, overall, the method yields small errors in all cases. This is a direct consequence of the approach's reliability and the MSE estimator optimality.

In addition, Fig. 4 presents visual results concerning our method performance on two textured images by showing the original, observed and reconstructed images. The blur and noise were eliminated, these results illustrating the method's ability to restore a significantly degraded textured image.

\section{CONCLUSION}

A fully Bayesian method for the threefold problem of estimating the textured image parameters, instrument parameters and hyperparameters has been presented. It also provides the deconvolved textured image, accurately restoring both textural content and intensity scale. To the best of our knowledge, no other method tackles this difficult problem. The Metropolis within Gibbs strategy provides posterior samples and their mean converges to the expectation. Moreover, the PM estimator guarantees that, at least from the MSE viewpoint, it cannot be outperformed by any concurrent method.

In addition, to aid in the comprehension of the problem and of the numerical results, we also presented a detailed analysis of the Fisher information. 


\section{REFERENCES}

[1] J.-L. Starck, E. Pantin, and F. Murtagh, "Deconvolution in astronomy: A review," Publ. Astron. Soc. Pacific, vol. 114, no. 800, pp. 1051-1069, 2002.

[2] P. Campisi and K. Egiazarian, Blind image deconvolution: theory and applications, CRC Press, 2007.

[3] S.D. Babacan, R. Molina, and A.K. Katsaggelos, "Variational Bayesian Blind Deconvolution Using a Total Variation Prior," IEEE Trans. Image Process., vol. 18, pp. 12-26, 2009.

[4] D. Tzikas, A. Likas, and N. Galatsanos, "Variational Bayesian sparse kernel-based blind image deconvolution with Student's-T priors," IEEE Trans. Image Process., vol. 18, no. 4, pp. 753-764, 2009.

[5] C. Vonesch and M. Unser, "A fast thresholded Landweber algorithm for wavelet regularized multidimensional deconvolution," IEEE Trans. Image Process, pp. 539549, 2008.

[6] M. Figueiredo and J.M. Bioucas-Dias, "Restoration of poissonian images using alternating direction optimization," IEEE Trans. Image Process., vol. 19, no. 12, pp. 3133-3145, 2010.

[7] F. Orieux, J.-F. Giovannelli, and T. Rodet, "Bayesian estimation of regularization and point spread function parameters for Wiener-Hunt deconvolution," Journal of Optical Society of America A, vol. 27, no. 7, pp. 1593-1607, 2010.

[8] G.R. Cross and A.K. Jain, "Markov random fields texture models," IEEE Trans. Pattern Anal. Mach. Intell., vol. PAMI-5, no. 1, pp. 25-39, 1983.

[9] G.L. Gimel'farb, Image Textures and Gibbs Random Fields, Kluwer Academic Publishers, 1999.

[10] T.E. Bishop, S.D. Babacan, B. Amizic, A.K. Katsaggelos, T. Chan, and R. Molina, Blind image deconvolution: theory and applications, chapter Blind Image Deconvolution: Problem Formulation and Existing Approaches, In [2], 2007.

[11] J.-M. Conan, L.M. Mugnier, T. Fusco, V. Michau, and G. Rousset, "Myopic deconvolution of adaptive optics images by use of object and point-spread function power spectra," Appl. Opt., vol. 37, no. 21, pp. 46144622, 1998.

[12] F. Orieux, J.-F. Giovannelli, T. Rodet, and A. Abergel, "Estimating hyperparameters and instrument parameters in regularized inversion. illustration for spire/herschel map making," Astronomy \& Astrophisics, vol. 549, pp. A83, 2012.

[13] J.-A. Conchello and Q. Yu, "Parametric blind deconvolution of fluorescence microscopy images: preliminary results," in Proc. SPIE, 1996, vol. 2655, pp. 164-174.

[14] J. Markham and J.-A. Conchello, "Parametric blind deconvolution of microscopic images: further results," in Proc. SPIE, 1998, vol. 3261, pp. 38-49.

[15] P. Pankajakshan, B. Zhang, L. Blanc-Feraud, Z. Kam,
J.C. Olivo-Marin, and J. Zerubia, "Parametric Blind Deconvolution for Confocal Laser Scanning Microscopy," in Int. Conf. of the IEEE Eng. in Med. and Bio. Soc., 2007, pp. 6531-6534.

[16] L. Tierney, "Markov chains for exploring posterior distributions," Ann. Stat., vol. 22, pp. 1701-1762, 1994.

[17] A. Gelman, G.O. Roberts, and W.R. Gilks, Bayesian Statistics 5, Oxford University Press, 1996. 